\title{
Formación inicial de los maestros y las maestras de primaria en derechos humanos en España
}

\author{
Initial Training of Primary School Teachers in the Field of Human Rights in Spain
}

\section{Formação inicial do corpo docente do ensino fundamental em direitos humanos na Espanha}

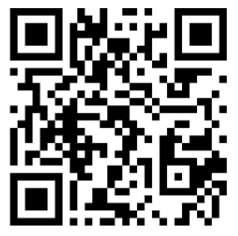

\section{Resumen:}

Objetivo. La siguiente investigación tiene como objetivo conocer qué cursos de las universidades españolas desarrollan los derechos humanos en la formación de los futuros maestros y maestras de Educación Primaria en derechos humanos. Metodología. Para ello, se han analizado 54 guías docentes de 46 universidades españolas que ofertaron durante el año 2018-2019 el Grado de Magisterio de Educación Primaria en castellano u otra lengua cooficial del territorio español mediante el análisis documental, apoyándonos en el software IBM SPSS Statistics 26 mediante análisis descriptivos. Resultados. Los resultados indican que: (i) cerca del 65\% de las universidades españolas tienen algún contenido sobre derechos humanos en los programas de estudios, (ii) la mayoría impartidos en asignaturas generales; (iii) y que la competencia más presente en los planes de estudio es que el profesorado sea capaz de diseñar y regular espacios para promover los derechos humanos. Conclusiones. A partir de estos resultados podemos inferir que estos contenidos tienen escasa presencia y relevancia. Además, la visión que se transmite de ellos es principalmente positivista y deja de lado temas como el interés superior del menor y la dignidad humana. En definitiva, es preciso repensar la formación inicial de los maestros y las maestras para que sean capaces de atender a los grandes desafíos humanitarios actuales y futuros.

Palabras claves: Derechos humanos; formación preparatoria de docentes; España; educación primaria; análisis documental. 
http://doi.org/10.15359/ree.26-1.16

http://www.una.ac.cr/educare

educare@una.ac.cr

\begin{abstract}
:
Objective. This research has, as a general objective, to know how Spanish universities train future teachers of Primary Education in the field of human rights. Method. For this and based on the documentary analysis approach and the software IBM SPSS Statistics 26, we have analyzed 54 teaching guides of 46 Spanish universities that offered the Primary Education Teaching Degree programs during 2018-2019 in Spanish or in another co-official language. Results. The results show that (i) about $65 \%$ of Spanish universities have some human rights content in the curricula, (ii) the majority taught in general subjects, (iii) and that the most present competence in the curricula is designing and regulating spaces to promote human rights. Conclusions. From these results, we can infer that these contents have a low presence and relevance in Primary Education Teaching Degree programs in Spain. In addition, the vision conveyed from them is mainly positivist and ignores essential issues such as the best interests of the child and human dignity. In short, it is necessary to rethink the initial training of teachers to enable them to face the great humanitarian challenges of the present and the future.
\end{abstract}

Keywords: Human Rights; preservice teacher education; Spain; primary education; documentary analysis.

\begin{abstract}
Resumo:
Introdução. Esta pesquisa tem como objetivo geral saber como as universidades espanholas capacitam futuros professores do ensino fundamental no campo dos direitos humanos. Metodologia. Para tal, foram analisados 54 guias pedagógicos de 46 universidades espanholas que ofereceram durante o ano 2018-2019 o Grau de Ensino Básico em espanhol ou outra língua cooficial do território espanhol através da análise documentária, apoiada pelo software IBM SPSS Statistics 26 por análise descritiva. Resultados. Os resultados indicam que: (i) cerca de $65 \%$ das universidades espanholas têm algum conteúdo em direitos humanos nos currículos, (ii) a maioria é ministrada em disciplinas gerais; (iii) e que a competência mais usada nos currículos é projetar e regular espaços para promover os direitos humanos. Conclusão. A partir desses resultados, podemos inferir que esses conteúdos apresentam baixa presença e relevância nas séries do Ensino Fundamental na Espanha. Além disso, a visão que é transmitida a partir deles é principalmente positivista e ignora questões essenciais como os melhores interesses da criança e a dignidade humana. Em suma, é necessário repensar a formação inicial do corpo docente, para tornálos capazes de enfrentar os grandes desafios humanitários do presente e do futuro.
\end{abstract}

Palavras-chave: Direitos humanos; formação de professores; Espanha; ensino fundamental; análise documental.

\title{
Pertinencia de formar al profesorado en derechos humanos: barbarie global, europea y española
}

En los últimos años la figura del profesorado ha emanado como un factor clave para alcanzar una educación de calidad. Un punto de inflexión fue el informe McKinsey de 2007 (Escudero Muñoz et al., 2017; González-Gil et al., 2016), en el que se concluía que era el factor más decisivo y diferenciador en los mejores sistemas educativos del mundo, pues allí donde los profesores eran de mayor calidad, sus estudiantes aprendían más (González-Gil et al., 2016). Pese 
http://doi.org/10.15359/ree.26-1.16

a la importancia innegable de dicho informe, Escudero Muñoz et al. (2017) critican duramente afirmaciones tan categóricas como la anterior, dado que pueden resultar "simplistas" (p. 84), ya que también se ha demostrado que los contextos socioculturales son factores muy influyentes en el éxito escolar del alumnado. Estos autores (Escudero Muñoz et al., 2017) van más allá, y se atreven -con gran acierto- a cuestionarse por qué es peligroso aceptar estas conclusiones tan absolutas. Por un lado, los innumerables factores sociopolíticos, estructurales, ideológicos y organizativos que influyen en la educación y en el docente y la docente tienen unas dimensiones y complejidad que las alejan de la intervención social y política. No obstante, si atribuimos toda la responsabilidad al cuerpo docente, este será mucho más accesible para cargarle con todo el peso de la educación de nuestros hijos y nuestras hijas. En cierto modo, el docente y la docente son seres vulnerables ante toda la presión que sostiene sobre sus cada vez más endebles y cuestionadas patas. En este contexto, el profesorado se siente incapaz e inseguro a la hora de trabajar en esta realidad tan dinámica y compleja, por lo que su formación inicial destaca como el elemento clave para una buena praxis y planificación docente (González-Gil et al., 2016).

Asimismo, parece haber consenso en la comunidad académica y educativa de que la figura del profesor es uno de los elementos más trascendentales en la calidad educativa (Escudero Muñoz et al., 2018). Ros-Garrido y García-Rubio (2016) reflexionan acerca de que la calidad docente es un concepto dinámico y sujeto al contexto sociohistórico donde se desarrolle su acción educativa, por lo que algo que "está bien hoy puede quedar obsoleto en un año o en tres meses, dada la celeridad de los cambios sociales en la actualidad" (Casanova, 2012, p. 9). Por ese motivo, Ros-Garrido y García-Rubio (2016) describen tres características fundamentales que debe tener hoy en día un buen docente:

- Debe ser capaz de garantizar la equidad, es decir, todo alumnado ha de adquirir unos aprendizajes mínimos.

- Ha de disponer de las competencias necesarias para diseñar e implementar una metodología flexible y pertinente, enfatizando en la adaptación de los contenidos curriculares a la diversidad del aula.

- Finalmente, el docente y la docente deben seleccionar los contenidos más relevantes y significativos para sus estudiantes.

El profesorado que adquiera y desarrolle estas competencias se convertirá en un poderoso agente en la lucha por la igualdad de oportunidades. Cobos (2014) no solo reafirma la importancia del profesorado en la calidad educativa, además:

Otorga la llave para mejorar el futuro del alumnado, y cuando comparte el conocimiento y se le facilita formación, al transmitir aprendizajes y valores universales fomenta la creación de ciudadanos y ciudadanas activos que comprenden los principios democráticos, promueven la tolerancia y participan en la sociedad en la que viven de forma responsable. (p. 8) 
http://doi.org/10.15359/ree.26-1.16

http://www.una.ac.cr/educare

educare@una.ac.cr

Sin embargo, para que esa transformación sea posible, el profesorado debe deconstruir sus creencias y saberes más profundos, pues ellos son los últimos responsables del proceso de enseñanza-aprendizaje, ya que se convierten en "director y ejecutor, de toda reforma propugnada desde la Administración Educativa" (Grau Company et al., 2009, p. 7). En ese nuevo camino por recorrer, el docente y la docente han de conformar reflexivamente una serie actitudes, conocimientos y destrezas que permitan mejorar la vida de sus estudiantes (Cobos, 2014). No es suficiente con cambios superficiales y legislativos, el cuerpo docente debe hacer suyo el proyecto educativo que compartirá con su alumnado, con las familias, con el centro educativo, con la sociedad y con la Humanidad. La formación inicial docente es mucho más que la adquisición de unos contenidos cognitivos, es desarrollar un compromiso, una sensibilidad y una actitud crítica que te permita llevar a cabo una praxis comprometida socialmente (García Gómez y Arroyo González, 2014). Aquí destaca la educación intercultural y reconstruccionista social, pues amplía la acción educativa del profesorado a toda la comunidad, de modo que se fortalece la "ciudadanía activa y democráticamente participativa" (Jiménez y Fardella, 2015, p. 424).

Antes de comenzar a reflexionar sobre hacia dónde se deben ir orientado los cambios -si es que son convenientes- en la formación inicial del profesorado, es preciso conocer cuál es la realidad en la que hoy vivimos en la Tierra, en Europa y en España, para comprender en mayor profundidad los sucesos que alertan sobre la necesidad de mejorar la formación inicial en materia de derechos humanos a los futuros maestros y maestras de Educación Primaria en España.

Mediavilla (2018) afirmó que en el año 2018 existían 33 conflictos armados en todo el mundo. Sin embargo, el Alto Comisionado de las Naciones Unidas para los Refugiados (ACNUR, 2020) limita a seis las guerras existentes, siendo estas las que tienen lugar en Yemen, Irak, Siria, Sudán del Sur, Somalia y Afganistán. Una de las cifras más alarmantes y cruentas de estos conflictos es que actualmente se estima que participan unos 300000 niños y niñas soldado en ellos (United Nations International Children's Emergency Fund [UNICEF], 2019). Lejos de las guerras y conflictos armados, la violencia en las calles y en el día a día sigue muy presente. Muestra de ello es que el Banco Mundial (2017) afirma que en el mundo hubo una media de 5,3 homicidios por cada 100000 habitantes en 2015, lo que en términos absolutos se traduce en 389049 homicidios en todo el mundo en ese mismo año.

Por otro lado, las mujeres son las principales víctimas de la violencia sexual. ONU Mujeres (2018) estima que alrededor del 35\% de las mujeres en el mundo han sufrido violencia sexual por parte de su pareja y 87000 mujeres fueron asesinadas en todo el mundo. En concreto, en cuanto a la violencia sexual, el $71 \%$ de las víctimas de trata de seres humanos son mujeres, de las cuales, tres de cada cuatro son niñas. No obstante, la mayoría de ellas -tres de cada cuatroson explotadas con fines sexuales. Por último, 650 millones de niñas fueron obligadas a casarse antes de alcanzar la mayoría de edad, aparte de las 200 que fueron víctimas de la mutilación genital femenina. 
http://doi.org/10.15359/ree.26-1.16

Si centramos el foco en el continente europeo, probablemente haya tres indicadores que reflejen fielmente la gravedad de la crisis humanitaria que asola al continente: el número de solicitudes de asilo, el número de muertes en el Mediterráneo y el auge de la nueva extrema derecha europea. En primer lugar, la Unión Europea ha recibido más de cuatro millones de solicitudes de asilo desde 2014, aunque 2015 fue el año con el número más alto de su historia, cuando se registró el número más alto de su historia (1 256 600) (Eurostat Statistics Explained [eurostat], 2021). La otra cara de la moneda -o la moneda perdida- son todas las personas que han perdido sus vidas en el Mar Mediterráneo. Así, la Organización Internacional para las Migraciones (OIM, 2018) contabilizó en los dos últimos años -2017 y 2018- cerca de 1600 muertes de personas migrantes en el Mediterráneo intentando alcanzar las costas europeas. Por otro lado, la presencia de los partidos de la nueva extrema derecha europea se ha incrementado en estas últimas elecciones europeas, pues tienen un total de 172 eurodiputados, lo que supone una representación parlamentaria del $23 \%$.

En España también encontramos estadísticas -personas- que reflejan cómo los derechos humanos son vulnerados sistemáticamente. La violencia de género es una muestra de ello. Así, gracias a la Delegación del Gobierno para la Violencia de Género (2019) sabemos que en 2018 el número de atención telefónica para las víctimas de esta violencia machista -016- fue de 73 464. Sin embargo, el número de denuncias interpuestas (166.961) fue mucho mayor que el de las Ilamadas. No obstante, los hechos más trágicos son los que se cobran víctimas humanas, un hecho que conocen muy de cerca los más 183 niños y niñas que se han quedado huérfanos desde el año 2015 tras el asesinato de 257 mujeres desde ese mismo año (Delegación del Gobierno para la Violencia de Género, 2019).

Por otro lado, la infancia, como colectivo potencialmente más vulnerable también sufre en su propia piel la realidad más lúgubre de nuestro tiempo. Save the Children (2018) denuncia que en España uno de cada tres menores se encuentra en riesgo de pobreza o exclusión social, lo que se traduce en que 2.610.370 de niños y niñas se encuentran al borde de una situación límite. Esta misma ONG (Save the Children, 2017) hace hincapié en que la protección de la infancia en las escuelas españolas tampoco está siendo garantizada, pues uno de cada 10 es víctima de algún tipo de acoso escolar, tomando paulatinamente más protagonismo el ciberbullying, siendo las niñas sus víctimas mayoritarias (65,6\%) (Ballesteros et al., 2018).

Ante semejante escenario, mejorar la formación inicial en materia de derechos humanos es de vital importancia. Pese a que en los últimos años se han hecho grandes esfuerzos, "todavía queda mucha tarea por hacer en esta cuestión tan trascendental" (Ros-Garrido y García-Rubio, 2016, p. 106), pues no debemos olvidar que la finalidad ulterior de la formación de los futuros maestros y maestras es mejorar el proceso de aprendizaje de su alumnado (Machado Pérez, 2015). Para ello, es preciso abandonar modelos de formación donde el profesorado sea un mero transmisor de conocimientos (Grau Company et al., 2009), porque tal y como defiende fervientemente Cobos 
http://doi.org/10.15359/ree.26-1.16

http://www.una.ac.cr/educare

educare@una.ac.cr

(2014), los conocimientos y competencias que adquiera el profesorado durante su formación son el factor más decisivo para asegurar un sistema educativo de calidad y, por ende, para combatir la pobreza y las desigualdades sociales. Sin embargo, esta tarea es tan importante como compleja (Marina et al., 2015), por lo que urge desarrollar programas de formación inicial que respondan a las necesidades sociales, políticas y económicas del presente y del futuro.

Finalmente, como señala Ramos Sánchez (2008), no debemos perder de vista las finalidades de la educación, reflejadas en la Ley Orgánica 2/2006, de 3 de mayo, de Educación, modificada por la Ley Orgánica 8/2013, de 9 de diciembre, para la mejora y la calidad educativa (Jefatura del Estado, 2013), que están estrechamente relacionadas con conocer, garantizar y promover los derechos humanos en todas las etapas educativas de la educación formal en España. Tan solo a través de un profesorado comprometido con los derechos humanos se podrá alcanzar una verdadera equidad socioeducativa (Casanova, 2012), pues el alumnado más necesitado, el excluido, los otros, son los que más sufren la falta de competencias por parte de los docentes y las docentes para paliar sus desigualdades (Novoa Palacios et al., 2019), y eso solo será posible, con una formación inicial desde y para los derechos humanos.

\section{Objetivos}

Esta investigación tiene como objetivo general conocer qué cursos de las universidades españolas desarrollan los derechos humanos en la formación de los futuros maestros y maestras de Educación Primaria en derechos humanos. Así, se establecieron tres objetivos específicos para dar respuesta a la pregunta de investigación, que son:

1. Describir la formación en derechos humanos en los planes de estudios de las universidades españoles en el Grado de Magisterio de Educación Primaria.

2. Analizar cómo se organizan las asignaturas y los contenidos en materia de derechos humanos en el Grado de Magisterio de Educación Primaria en España.

3. Discutir sobre si los planes de estudio de las universidades españolas responden a las necesidades educativas, sociales, económicas y políticas en materia de derechos humanos.

\section{Diseño metodológico}

El enfoque metodológico adoptado para esta investigación es el análisis documental, ya que permite categorizar, analizar y posteriormente, elaborar propuestas de mejora y conocimiento a partir de los documentos analizados (Hernández-Ayala y Tobón-Tobón, 2016; Jiménez et al., 2017). Por otra parte, se ha empleado el software de análisis cuantitativo IBM SPSS Statistics 26 para sistematizar la información. Además, esto permitió realizar diferentes análisis descriptivos de frecuencias. 
http://doi.org/10.15359/ree.26-1.16

Una de las primeras decisiones que se tomaron a la hora de diseñar la investigación fue sobre en qué documentos institucionales se recogería la información requerida, en otras palabras, cuáles serían las unidades de análisis. Finalmente, se analizaron todas las guías docentes de las asignaturas del grado de Magisterio en Educación Primaria de 46 universidades españolas presentes en sus páginas web. Por un lado, estos documentos suponen un compromiso mutuo entre la institución y el alumnado sobre las normas, los criterios de evaluación, los contenidos o las competencias que se desarrollarán en la asignatura. Además, más allá de las competencias generales que el título promueva, resulta especialmente relevante dilucidar en qué asignaturas y qué peso tienen los contenidos de derechos humanos en la formación inicial del maestro de Educación Primaria, y eso se observa con nitidez en dichos documentos.

Para recopilar y organizar la información de forma sistemática se elaboró un instrumento específico, que consta de cuatro categorías de análisis: 1) institución; 2) organización; 3) contenidos; y 4) competencias. La primera permitió identificar la universidad, en qué Comunidad Autónoma se encuentra y su titularidad pública o privada. Por otro lado, en cuanto a la categoría "organización" se clasificó el carácter de la asignatura (obligatoria, optativa o general), en qué curso se imparte, su duración y el tipo de asignatura (específica o general) con relación a los derechos humanos. En este sentido, una asignatura es específica cuando todos sus contenidos se abordan desde un enfoque de derechos humanos, mientras que las asignaturas generales son aquellas que dedican una parte de sus contenidos a esta cuestión. Respecto a la organización, también es importante conocer qué departamentos universitarios se ocupan de estas asignaturas y contenidos.

La tercera categoría es la referida a los contenidos, para lo que en primer lugar es necesario conocer si tienen contenidos sobre ello o no. Asimismo, se diferencia entre bloques de contenidos específicos o contenidos integrados en un bloque de contenidos general. La cuarta categoría se ocupa de identificar las competencias y los resultados de aprendizaje que desarrollan cada universidad. Para terminar, se han agrupado distintas respuestas en las variables de asignaturas, de departamento, de contenidos y de competencias para facilitar la comprensión de los datos.

Por otra parte, es preciso señalar los criterios utilizados para buscar y seleccionar los datos. Dicho proceso de búsqueda documental en las páginas web institucionales de las universidades se produjo entre septiembre y noviembre de 2019. En este estudio se han analizado 54 guías docentes de 46 universidades españolas que ofertaron durante el año 2018-2019 el Grado de Educación Primaria en castellano o en otra lengua cooficial del territorio español. De ellas, 37 son de titularidad pública, lo que supone el $80,43 \%$ del total. Por otro lado, las nueve restantes son privadas, por lo que la financiación corre a cargo de particulares.

Estas universidades se sitúan en 16 Comunidades Autónomas (Andalucía, Aragón, Principado de Asturias, Cantabria, Castilla-La Mancha, Extremadura, Islas Baleares, La Rioja, Canarias, Región de Murcia, Galicia, País Vasco, Castilla y León, Comunidad Valenciana, Cataluña y Comunidad de 
http://doi.org/10.15359/ree.26-1.16

http://www.una.ac.cr/educare

educare@una.ac.cr

Madrid). Así, su distribución es distinta a lo largo del territorio nacional. La Comunidad de Madrid y Andalucía son las regiones con más universidades (7), mientras que Aragón, el Principado de Asturias, Cantabria, Extremadura e Islas Baleares solo tienen una cada una.

Respecto a la presencia de universidades privadas en las distintas comunidades, es preciso destacar la Comunidad de Madrid, pues es donde mayor presencia tienen, con un total de tres. Esta Comunidad es seguida de cerca por Cataluña, quien tiene dos universidades privadas. Además, la Región de Murcia, la Comunidad Valenciana, País Vasco y Castilla y León son las otras regiones que tienen alguna universidad privada que oferte el Grado de Magisterio en Educación Primaria.

\section{Resultados}

\section{Asignaturas en las que se imparten derechos humanos}

En primer lugar, se ha podido discernir cómo dos universidades -una andaluza y otra madrileña- no tenían ninguna referencia a la formación en derechos humanos de sus estudiantes, mientras que las 44 universidades restantes sí. De tal modo, se puede afirmar que prácticamente todas las universidades españolas forman, como se mostrará a continuación, a su alumnado del Grado de Magisterio de Educación Primaria en derechos humanos.

Por otro lado, 22 universidades, es decir, cerca del 50\% de ellas, no tiene ningún contenido general ni específico sobre esta cuestión. Esto sucede en Aragón, el Principado de Asturias, Extremadura, Canarias, Castilla y León (en 2 universidades), Andalucía (3 universidades), País Vasco (3 universidades), Comunidad Valenciana (3 universidades), Cataluña (3 universidades) y la Comunidad de Madrid (4 universidades). Sin embargo, en las 22 universidades que disponen de contenidos sobre esta cuestión se han identificado 32 guías docentes, o asignaturas, donde se aborda este tema. No obstante, la atención es diferente. La mayoría de las universidades (43,8\%) presenta estos contenidos en bloques específicos. Por otro lado, el 34,4\% (11 universidades) lo presentan en bloques temáticos. Para terminar, el 21,9\% de las universidades aboga por presentar, trabajar y analizar los contenidos desde un enfoque global de derechos humanos.

Las 32 asignaturas en las que están presentes los contenidos en derechos humanos se pueden diferenciar entre específicas o generales. Las primeras son aquellas que la gran mayoría de sus contenidos hacen alusión a los derechos humanos, o bien, trabajan desde un enfoque global de derechos. Así, podemos observar que existen cinco asignaturas que cumplen estos requisitos. De tal modo, las asignaturas se han agrupado en dos propuestas: Educación para la paz, la ciudadanía y los derechos humanos y Accesibilidad y discapacidad. La primera de ellas está presente en tres planes de estudio (Universidad de Málaga, Universidad de Valladolid y Universidad de Salamanca), mientras que la de Accesibilidad y discapacidad la podemos estudiar tan solo en la Universidad de Murcia y la Universidad de La Rioja. Además, esta última asignatura tiene la peculiaridad de que es optativa en las dos universidades en las que se imparte, y su carga lectiva es de 3 ECTS. Mientras tanto,

8 
http://doi.org/10.15359/ree.26-1.16

Educación para la paz, la ciudadanía y los derechos humanos puede ser obligatoria (2 universidades), básica (1 universidades) u optativa (1 universidad). No obstante, tan solo la asignatura optativa en la Universidad de la Rioja tiene 4,5 ECTS, pues las demás le otorgan 6 ECTS.

Por otro lado, si atendemos a las asignaturas genéricas en las que se imparten contenidos de derechos humanos, encontramos un gran abanico de posibilidades, pues estos se desarrollan en 10 asignaturas. Las más frecuentes en las que se trabajan estos contenidos son: Sociedad, escuela, educación, religión y familia, Teoría, política e historia de la educación y Educación para la igualdad y la ciudadanía. En estas tres asignaturas genéricas se aglutina el 55,5\% de las materias que desarrollan los derechos humanos. Por otrolado, Didáctica y Organización Escolary Educación inclusiva y atención a la diversidad también ocupan un papel relevante en esta formación, ya que seis asignaturas integran estos contenidos en sus guías docentes. No obstante, el resto de las materias tienen una presencia mucho menor, pues hay asignaturas muy variadas que abordan esta cuestión en sus planes de estudio de forma aislada a nivel nacional.

Estas materias son principalmente de carácter básico $(77,8 \%)$. Solo dos asignaturas obligatorias tienen 6 ECTS, aunque resulta relevante que existan cuatro asignaturas optativas $(14,8 \%)$ para mejorar la formación inicial del alumnado en materia de derechos humanos. No obstante, el carácter de la asignatura parece no influir significativamente en su duración, aunque bien es cierto que las básicas tienen una media de 6,86 ECTS y las optativas de 5,25 ECTS.

Por otro lado, el 50\% de las asignaturas en materia de derechos humanos tienen lugar durante el primer curso, preferentemente en el cuatrimestre inicial (10 universidades). La siguiente opción más frecuente es la de impartir estos contenidos en segundo curso (25\%), aunque en esta ocasión se suelen trabajar en el último cuatrimestre (5 universidades). No obstante, la organización de las asignaturas que se desarrollan en tercero y cuarto son muy similares. Para terminar, es preciso recalcar que la Universidad de Castilla-La Mancha, la Universidad Autónoma de Barcelona y la Universitat de Rovira i Virgili son las únicas en dotar a estas asignaturas de una duración anual. Otra cuestión relevante es conocer qué departamentos son los responsables de enseñar estos contenidos. Este análisis nos permitió observar cómo los departamentos de Didáctica de las Ciencias Humanas y Filosofía (25\%) y Didáctica y Organización Escolar $(21,9 \%)$ son los principales encargados de esta labor. No obstante, Educación (15,6\%) y Ciencias políticas, ética y sociología (15,6\%) también tienen una presencia notable, mientras que Pedagogía $(12,5 \%)$ y, especialmente, Teoría, didáctica e historia de la educación $(9,4 \%)$ disponen de menos asignaturas que desarrollen estos contenidos.

\section{La selección de contenidos para la enseñanza de derechos humanos}

Por otro lado, cómo se organizan y qué tipo de contenidos se enseñan durante la formación inicial de los futuros maestros es una cuestión clave. Así, hemos podido distinguir dos formas de organizar los contenidos: en bloques generales y en bloques específicos. Los genéricos son 
http://doi.org/10.15359/ree.26-1.16

http://www.una.ac.cr/educare

educare@una.ac.cr

aquellos en los que el peso de los derechos humanos no es prioritario, son un mero contenido más. Mientras tanto, los específicos giran en torno a ellos. En primer lugar, el 40,62\% de los contenidos se integran dentro de los bloques generales, de modo que las universidades españolas optan por abordar los contenidos en derechos humanos de forma específica.

Por ello, hemos identificado seis grandes bloques generales, aunque en Psicología de las personas con discapacidad, La enseñanza religiosa en la escuela y La educación como política pública y el papel del Estado (Derecho a la educación y obligatoriedad escolar, su presencia es meramente anecdótica, pues solo se trabaja aborda en una ocasión). Por otro lado, los bloques de Convivencia social, educación, ciudadanía y derechos humanos y el de Aspectos sociopolíticos de la comunidad escolar y del currículo integran estos contenidos en dos ocasiones cada uno. Sin embargo, el bloque hegemónico es el que aborda el derecho a la educación y el papel del Estado, pues representa el 53,8\% de los contenidos generales.

En cuanto a los bloques específicos, 19 asignaturas apuestan por este modelo. Son pocas las universidades que dedican un bloque de contenidos específico a la conceptualización, a la fundamentación, la evolución histórica (1 universidad) y a la ética (1 universidad) de los derechos humanos. Resulta especialmente llamativo que esto se repita con los derechos de la infancia, pues a pesar de que tiene presencia de forma integral en un gran número de bloques de contenidos, tan solo la Universidad Pontificia de Salamanca dedica un bloque a toda esta temática.

No obstante, la Dignidad humana, los derechos humanos para la igualdady la justicia social se trabaja en tres asignaturas. Sin embargo, el 31,6\% de las materias tienen como objetivo enseñar al alumnado cómo desarrollar su labor docente acorde al enfoque de derechos humanos. Sin embargo, el bloque que tiene más relevancia es el del Papel de la escuela en la sociedad actual y su importancia con los derechos humanos y la ciudadanía democrática, pues es trabajado desde el $36,8 \%$ de las asignaturas.

\section{Competencias docentes en la formación inicial}

En las guías docentes se mencionan diferentes competencias que debe adquirir y desarrollar el alumnado durante las asignaturas y a lo largo del grado universitario. Tras analizar las 54 guías docentes, se identificaron 10 competencias comunes:

1. Respetary promover los derechosfundamentales recogidos en la legislación internacional en materia de derechos humanos mediante la cultura de paz y la democracia.

2. Promover el respeto y promoción del principio de no-discriminación y accesibilidad para las personas con diversidad funcional.

3. Justificar y gestionar las habilidades interpersonales en el clima escolar desde respetando los derechos humanos.

4. Desarrollar un compromiso ético con la educación por y para los derechos humanos. 
http://doi.org/10.15359/ree.26-1.16

http://www.una.ac.cr/educare educare@una.ac.cr

5. Conocer la sociología de los derechos humanos, de los valores democráticos y de la solidaridad.

6. Diseñar y regular espacios y actividades que promuevan los derechos humanos bajo los principios de universalidad y no-discriminación

7. Identificar vulneraciones de derechos humanos.

8. Promover los derechos humanos y la sostenibilidad del planeta desde la Didáctica de Ciencias Experimentales.

9. Desarrollar relaciones de respeto en sintonía con los derechos humanos en la educación física.

10. Conocer y respetar los derechos sexuales y reproductivos.

En primer lugar, hay que destacar que se han identificado 42 universidades que en sus guías docentes persiguen desarrollar competencias en materia de derechos humanos en su alumnado del Grado de Magisterio de Educación Primaria. La primera competencia mencionada es desarrollada por 11 universidades españolas, es decir, el 26,3\% de las que pretende que su alumnado adquiera una serie de competencias en esta cuestión. Por otro lado, la competencia de promover los derechos humanos de las personas con discapacidad tan solo está presente en cinco universidades $(11,6 \%)$. Resulta llamativa la escasa presencia que tiene la competencia de desarrollar habilidades interpersonales para mejorar las relaciones entre la comunidad educativa con base en los derechos humanos, pues apenas dos universidades recogen esta competencia en sus guías docentes. Asimismo, otras competencias apenas han sido recogidas en las guías docentes en una ocasión (competencia específica de Educación Física; competencia de Ciencias Experimentales; identificar obstáculos para garantizar los derechos humanos; y promover derechos sexuales). También es pertinente señalar el hecho de que tan solo cuatro universidades $(9,5 \%)$ estimen oportuno que su alumnado desarrolle la competencia de conocer el origen y la evolución histórica de los derechos humanos.

Sin embargo, una de las competencias más desarrolladas es que los futuros maestros adquieran un compromiso ético con los derechos humanos $(23,8 \%)$. En último lugar, la competencia que está presente en prácticamente todas las guías $(88,1 \%)$ es la de diseñar y regular espacios y actividades que promuevan los derechos humanos.

\section{Discusión y conclusiones}

Los resultados expuestos anteriormente invitan a reflexionar y extrapolar ciertas conclusiones sobre la formación inicial del estudiantado del Grado de Educación Primaria en España. La primera de ellas es que 42 universidades españolas disponen de competencias relacionadas con los derechos humanos en sus guías docentes. Sin embargo, a pesar de que el $65 \%$ de ellas cuenta con algún contenido sobre esta cuestión, ya sea de carácter genérico o 
http://doi.org/10.15359/ree.26-1.16

http://www.una.ac.cr/educare

educare@una.ac.cr

específico, cabe pensar en la posibilidad de que esto sea consecuencia del fuerte impacto del enfoque competencial en la universidad española. Por otra parte, también parece pertinente preguntarse si la escasa relevancia que tienen los derechos humanos en la formación inicial permite alcanzar los ambiciosos objetivos propuestos por la legislación nacional.

Las universidades españolas determinan qué competencias son más relevantes para sus estudiantes y eso se infiere por su presencia o ausencia en las guías docentes. En este sentido, resulta llamativo observar cómo existe un amplio consenso en que sean capaces de diseñar y regular actividades que fomenten los derechos humanos, pero apenas el 23,8\% plantea explícitamente que adquiera un compromiso ético con estos derechos. Si a ello le sumamos la falta de contenidos filosóficos, que obliguen a reflexionar críticamente al alumnado sobre el origen y la pertinencia de los derechos humanos, estaremos formando maestros tecnócratas, relegados a meros transmisores de conocimientos. Esto se observa de nuevo en que el $15 \%$ de los contenidos generales están relacionados con la organización escolar y el orden jurídico educativo. Así, el alumnado consolida una visión positivista de los derechos humanos, dejando de lado elementos imprescindibles a la hora de abordar estos contenidos, como son la dignidad humana y el interés superior del menor.

Por otro lado, una cuestión que hay que destacar es que tan solo dos universidades estimen necesario que sus estudiantes puedan desarrollar habilidades interpersonales $y$ acciones conjuntas a favor de los derechos humanos con la comunidad educativa. De esta manera, se transmite una visión de escuela hermética, totalmente desconectada de su realidad más cercana, renunciando de este modo a un gran número de recursos espaciales, humanos, afectivos y personales.

En definitiva, la formación inicial de los maestros y las maestras de Educación Primaria en las universidades españolas ha de intensificar sus contenidos en materia de derechos humanos. Esta apuesta por la igualdad, la libertad y la dignidad humana sitúa al docente como un poderoso agente de cambio social (Casanova, 2012; Cobos, 2014; Machado Pérez, 2015). Sin embargo, eso tan solo es posible si son capaces de comprender y atender a las demandas sociales presentes y futuras, algo que actualmente dista de ser lo ideal en temas como la crisis de refugiados, el cambio climático o los derechos de la infancia. Así, es imprescindible un cambio de paradigma donde el profesorado no sea un mero ejecutor de la Administración Educativa (Grau Company et al., 2009), si no que sea un intelectual crítico y reflexivo comprometido con la Humanidad (García Gómez y Arroyo González, 2014), porque como Marina et al. (2015) señalaban, formar docentes de calidad no es algo sencillo, pero sí es un motivo por lo que merece la pena luchar cada día.

\section{Agradecimiento}

Este trabajo ha contado con la colaboración de Juan Antonio Núñez Cortés, profesor de la Universidad Autónoma de Madrid, en la revisión de su redacción. 


\section{Declaración de Material complementario}

Este artículo tiene disponible, como material complementario:

-La versión preprint del artículo en https://doi.org/10.5281/zenodo.4891128

\section{Referencias}

Alto Comisionado de las Naciones Unidas para los Refugiados. ACNUR. (2020). Países en guerra actualmente en 2020. Autor. https://eacnur.org/blog/paises-en-guerra-actualmente-tc alt45664n o pstn o pst/

Ballesteros, B., Pérez de Viñaspre, S., Díaz, D. y Toledano, E. (2018). III estudio sobre acoso escolar y ciberbullying según los afectados. Fundación Mutua Madrileña; Fundación ANAR. https://www.anar.org/wp-content/uploads/2018/09/III-Estudio-sobre-acoso-escolar-yciberbullying-según-los-afectados.pdf

Banco Mundial. (2017). Datos de libre acceso del Banco Mundial [Base de datos]. https://datos. bancomundial.org

Casanova, M. A. (2012). El diseño curricular como factor de calidad educativa. REICE. Revista Iberoamericana sobre Calidad, Eficacia y Cambio en Educación, 10(4), 6-20. https://www. redalyc.org/pdf/551/55124841002.pdf

Cobos, M. (2014,). La formación docente es clave para la calidad educativa. Revista Crítica, (994), 8-11. http://www.revista-critica.com/archivo/49-las-migraciones-en-un-mundoglobalizado

Delegación del Gobierno para la Violencia de Género. (2019). Portal estadístico. http:// estadisticasviolenciagenero.igualdad.mpr.gob.es

Escudero Muñoz, J. M., Cutanda López, M. T., y Trillo Alonso, J. F. (2017). Aprendizaje docente y desarrollo profesional del profesorado. Profesorado. Revista de Currículum y Formación de Profesorado, 21(3), 83-102. https://www.redalyc.org/pdf/567/56752489005.pdf

Escudero Muñoz, J. M., González González, M. T. y Rodríguez Entrena, M. J. (2018). Los contenidos de la formación continuada del profesorado: ¿Qué docentes se están formando? Educación XX1, 21(1), 157-180. https://doi.org/10.5944/educXX1.20183

Eurostat Statistics Explained. (2021). Asylum statistics. https://ec.europa.eu/eurostat/statisticsexplained/index.php?title=Asylum statistics 
http://doi.org/10.15359/ree.26-1.16

http://www.una.ac.cr/educare

educare@una.ac.cr

García Gómez, L. y Arroyo González, M. J. (2014). La formación del profesorado en Educación Intercultural: Un repaso sobre su formación inicial y permanente. Revista de Educación Inclusiva, 7(2), 127-142. https://revistaeducacioninclusiva.es/index.php/REl/article/ view/153/147

Gónzalez-Gil, F., Martín-Pastor, E., Poy, R. y Jenaro, C. (2016). Percepciones del profesorado sobre la inclusión: Estudio preliminar. Revista Electrónica Interuniversitaria de Formación del Profesorado, 19(3), 11-24. https://doi.org/10.6018/reifop.19.3.219321

Grau Company, S., Gómez Lucas, C. y Perandones González, T. M. (2009). 1. La formación del profesorado como factor decisivo de la excelencia educativa. En C. Gómez Lucas y S. Grau Company (Coords.), Propuestas de diseño, desarrollo e innovaciones curriculares y metodología en el EEES (pp. 7-26). Universidad de Alicante. https://rua.ua.es/dspace/ bitstream/10045/13199/1/PROPUESTAS\%20CAP.\%201.pdf

Hernández-Ayala, H., y Tobón-Tobón, S. (2016). Análisis documental del proceso de inclusión en la educación. Ra Ximhai, 12(6), 399-420. https://doi.org/10.35197/rx.12.01.e3.2016.27.hh

Jefatura del Estado (10 de diciembre de 2013). Ley Orgánica 8/2013, de 9 de diciembre, para la mejora de la calidad educativa. Boletín Oficial del Estado, núm. 295, pp. 97858-97921. https://www.boe.es/eli/es/lo/2013/12/09/8

Jiménez, F., Aguilera Valdivia, M., Valdés Morales, R. y Hernández Yáñez, M. (2017). Migración y escuela: Análisis documental en torno a la incorporación de inmigrantes al sistema educativo chileno. Psicoperspectivas. Individuo y Sociedad, 16(1), 105-116. https://doi. org/10.5027/psicoperspectivas-vol16-issue1-fulltext-940

Jiménez, F. y Fardella, C. (2015). Diversidad y rol de la escuela: Discursos del profesorado en contextos educativos multiculturales en clave migratoria. Revista mexicana de investigación educativa, 20(65), 419-441. http://www.scielo.org.mx/scielo.php?pid=S1405$66662015000200005 \&$ script $=$ sci abstract\&tlng=en

Machado Pérez, E. M. (2015). La formación del profesorado. Revista Arista Digital, (54), 8-12. https://fundacionarista.es/project/numero-54-marzo-2015/

Marina, J. A., Pellicer, C. y Manso, J. (2015). Libro Blanco de la profesión docente y su entorno escolar. https://repositorio.uam.es/bitstream/handle/10486/669976/Libro_Marina_2015. pdf? sequence $=1$ \&isAllowed $=\mathrm{y}$

Mediavilla, M. (12 de enero, 2018). Más de 30 conflictos armados dejan un reguero de muerte [Mensaje en un blog]. Aministía Internacional. https://www.es.amnesty.org/en-queestamos/blog/historia/articulo/mas-de-30-conflictos-armados-dejan-un-reguero-demuerte/ 
http://doi.org/10.15359/ree.26-1.16

http://www.una.ac.cr/educare educare@una.ac.cr

Novoa Palacios, A., Pirela Morillo, J., e Inciarte González, A. (2019). Educación en y para la democracia. Utopía Y Praxis Latinoamericana, 24(3), 60-74. https://produccioncientificaluz. org/index.php/utopia/article/view/29686

ONU Mujeres. (2018). Hechos y cifras: Acabar con la violencia contra mujeres y niñas. http://www. oacnudh.org/hechos-y-cifras-acabar-con-la-violencia-contra-mujeres-y-ninas/

Organización Internacional para las Migraciones (OIM). (20 de abril de 2018). Se contabilizan 18.575 y 559 muertes en el Mediterráneo en 2018. https://www.iom.int/es/news/secontabilizan-18575-y-559-muertes-en-el-mediterraneo-en-2018

Ramos Sánchez, J. L. (2008). Reformas, investigación, innovación y calidad educativa. Revista Iberoamericana de Educación, 46(2), 1-12. https://doi.org/10.35362/rie4622002

Ros-Garrido, A., y García-Rubio, J. (2016). La calidad en la formación del profesorado del sistema educativo y de los certificados de profesionalidad. Edetania. Estudios y propuestas socioeducativas, (50), 101-119. https://revistas.ucv.es/index.php/Edetania/article/ view/21/21

Save the Children. (2017). Bullying o acoso escolar. https://www.savethechildren.es/donde/ espana/violencia-contra-la-infancia/acoso-escolar-bullying

Save the Children. (21 de junio de 2018). La pobreza infantil sigue por encima de los niveles previos a la crisis. https://www.savethechildren.es/actualidad/la-pobreza-infantil-sigue-porencima-de-los-niveles-previos-la-crisis

United Nations International Children's Emergency Fund. (2019). Niños soldado. Cada 12 de febrero se conmemora el Día Internacional contra el Uso de Niños Soldado. https://www. unicef.es/ninos-soldado 